Running head: MINDFULNESS AND PROBLEM GAMBLING

Mindfulness and problem gambling: A review of the literature

\author{
Steven M. de Lisle ${ }^{1}$, Nicki A. Dowling ${ }^{2}$ \\ \& J. Sabura Allen ${ }^{1}$
}

Corresponding Author:

Nicki A. Dowling, Problem Gambling Research and Treatment Centre, Melbourne Graduate School of Education, Level 5, 100 Leicester Street, University of Melbourne, Victoria, Australia 3010

Email: n.dowling@unimelb.edu.au 


\footnotetext{
${ }^{1}$ School of Psychology and Psychiatry, Monash University, Melbourne, Victoria, Australia

${ }^{2}$ Problem Gambling Research and Treatment Centre, University of Melbourne, Victoria, Australia
}

\begin{abstract}
This article reviews the literature with respect to mindfulness and its potential for reducing the severity of problem gambling behaviour. Interest in the role of mindfulness as a treatment for problem gambling has gained the attention of researchers across Australia, the United States, and Canada. However, the literature is limited and current studies have severe methodological limitations. Despite this issue, investigations have revealed that dispositional mindfulness is related to less severe problem gambling outcomes and that psychological distress, overconfidence and risk willingness, myopic focus on reward and ego involvement may act as mediators in this relationship. Moreover, the literature indicates that the inverse relationship between dispositional mindfulness and psychological distress may be mediated by factors such as values clarification; emotional, cognitive, and behavioural flexibility; nonattachment; emotion dysregulation/distress intolerance; thought suppression; and rumination. This article discusses the theoretical and clinical implications of these relationships with respect to mindfulness-based interventions for problem gambling. It is recommended that the approach be considered with cautious optimism.
\end{abstract}

\title{
Keywords
}

Problem gambling, gambling, mindfulness, treatment, intervention 
Currently, cognitive-behavioural therapies (CBT) are cautiously recommended as "best practice" for the psychological treatment of problem gambling (Ladouceur, Sylvain, Boutin, \& Doucet, 1998; López Viets \& Miller, 1997; Problem Gambling Research and Treatment Centre, 2011; Rickwood, Blaszczynski, Delfabbro, Dowling, \& Heading, 2010). However, CBT does not work for all problem gamblers (López Viets \& Miller, 1997; Westphal, 2008), highlighting the need for innovative treatment approaches that can enhance treatment effectiveness. Mindfulness-based interventions represent a 'third wave' that expands upon the CBT tradition by incorporating eastern approaches to the mind and body within a cognitive-behavioural framework (Hayes, Follette, \& Linehan, 2004; Williams \& Swales, 2004). A number of studies have already been conducted on the efficacy of mindfulness-based psychotherapy in the treatment of a wide range of diverse conditions, including substance abuse, chronic pain, stress, anxiety, depression, eating disorders, and borderline personality disorder (Baer, 2003; Kabat-Zinn, 2005; Linehan, 1993a, 1993b; Segal, Williams, \& Teasdale, 2002). There is now research interest in the role of mindfulness in the treatment of problem gambling in order to improve problem gambling outcomes (de Lisle, Dowling, \& Allen, 2011a, 2011b; Lakey, Campbell, Brown, \& Goodie, 2007; Toneatto, Vettese, \& Nguyen, 2007). This article reviews the literature to consider the potential of mindfulness-based psychotherapy for problem gambling and the mechanisms by which mindfulness may be therapeutically beneficial.

\section{The Role of Mindfulness in Problem Gambling}

Mindfulness is defined as "the process of observing body and mind intentionally, of letting ... experiences unfold from moment to moment and accepting them as they are" (Kabat-Zinn, 2005, p.23). In an effort to facilitate empirical investigations into mindfulness, a consensus panel was convened to provide an operational definition of the mindfulness 
construct (Bishop et al., 2004). The panel derived a definition that comprised of two components: self-regulation and orientation to experience.

According to Bishop et al. (2004), self-regulation "involves sustained attention, attention switching, and the inhibition of elaborative processing" (p.233). Thus, a gambler with a higher degree of mindfulness should be able to sustain an awareness of gamblingrelated thoughts and feelings for a prolonged period of time. From this position, attention can then be switched from whatever is noticed to an alternative perspective. This perspective can represent any object to which attention can be turned once an individual notices that they have become distracted by a gambling stimulus. Bishop et al. considered this process helped to inhibit secondary elaborative processing of the thoughts, feelings and sensations involved in stimulus selection. Gamblers at the problematic end of the gambling continuum tend to report numerous gambling-related cognitive distortions and biases that are unique to this group (Joukhador, Maccallum, \& Blaszczynski, 2003). So rather than experiencing the present moment through a filter of beliefs, assumptions and expectations about gambling, the ability of more mindful gamblers to self-regulate may help provide a wider perspective from which to view their gambling behaviour, thereby providing greater choice about what action to perform.

The other component is orientation to experience. Orientation to experience refers to the adoption of a sense of curiosity, openness and acceptance of present moment experience (Bishop et al., 2004). For problem gamblers with a higher level of mindfulness, this ability would afford the opportunity to dispassionately observe thoughts, feelings, and sensations come and go in the present moment. Bishop et al. considered that monitoring the stream of consciousness in this manner would allow individuals to see complex relationships between thoughts, feelings and sensations and to "discern the meanings and causes of experience and behaviour" (p. 234). For mindful gamblers, this capacity may result in the decision not to 
initiate or prolong a gambling episode even when confronted by highly arousing or distressing emotions. Taken together, mindfulness training may be helpful in promoting awareness of previously unattended thoughts, feelings and physical sensations that drive maladaptive behaviour (Krasner, 2004) and represent a possible pathway by which the severity of problem gambling outcomes may be reduced.

There is emerging evidence relating to the role of mindfulness in problem gambling (de Lisle, Dowling, \& Allen, 2011b; Lakey et al., 2007). In a non-randomised study of 185 undergraduate students who gambled at least weekly, Lakey et al. (2007) found that dispositional mindfulness was negatively related to gambling-related pathology after controlling for gambling frequency and trait self-control. In this context, dispositional mindfulness can be conceived as an innate skill or tendency that can contribute to the behavioural responses of a given situation through mechanisms such as greater self-control. In this way, Lakey et al. speculated that a higher level of dispositional mindfulness facilitated the development of greater present-centred awareness which then allowed for more adaptive behavioural choices. However, as the Lakey et al. study involved undergraduate students, these results can not be extended to the general population of treatment seeking problem gamblers. To address this issue, a study conducted by de Lisle et al. (2011b) found that two samples of treatment seeking problem gamblers displayed significantly lower levels of dispositional mindfulness than normative samples of adult community members and university students. In investigating whether dispositional mindfulness was related to gambling behaviour in treatment seeking populations of problem gamblers, this study found that dispositional mindfulness was negatively related to gambling urges, gambling preoccupation, problem gambling severity, gambling expenditure, and gambling frequency.

Both of these studies suggest that problem gambling outcomes may be improved by mindfulness training. However, only two case studies have reported on the use of 
mindfulness-based psychotherapy for problem gambling. The first was a case study conducted by Toneatto et al. (2007) of a male gambler 'in his sixties' who had not benefited from CBT. While CBT highlighted the cognitive distortions displayed by the participant in addition to providing him with both an intellectual understanding of the erroneous perceptions he held and an explanation of the deleterious consequences of maintaining his gambling, the participant failed to refrain from gambling. Over time, the participant began to use justification strategies such as “one more time won't hurt” and any intellectual understanding of cognitive distortions and psychosocial consequences of gambling was rapidly overwhelmed in favour of a resumption of gambling behaviour. After this, the participant agreed to participate in mindfulness training. This training taught the participant to observe what was happening in his mind and become aware of gambling-related thoughts and feelings with a sense of openness and curiosity. The result was a rapid dissolution of the urge to gamble. While gambling-related thoughts and feelings continued to occur, the intensity of these thoughts and feelings greatly diminished, allowing the subject to make more adaptive choices (Toneatto et al., 2007). However, no follow-up of the participant was reported, and detail of the mindfulness practice used was not specified.

The second study reported on a modified version of mindfulness-based cognitive therapy $(\mathrm{MBCT})$ in the treatment of a female problem gambler in her sixties who failed to fully abstain from gambling following traditional CBT (de Lisle, Dowling, \& Allen, 2011a). The subject refrained from gambling over the intervention phase and remained abstinent from gambling at a ten-week follow-up evaluation. Moreover, her depression and anxiety scores reduced to sub-clinical levels at the follow-up evaluation, suggesting that the intervention was helpful in reducing psychological distress. This study examined mindfulness at a facet level using the Five Factor Mindfulness Questionnaire (FFMQ; Baer, Smith, Hopkins, Krietemeyer, \& Toney, 2006) to gain more insight into the role of mindfulness in improving 
problem gambling outcomes. The facets are: (1) Observing - openness to experience and attending to internal and external stimuli; (2) Non-Judging - the ability to accept current experience; (3) Acting with Awareness - attention to current activity and avoiding automatic pilot; (4) Describing - the ability to recognise and label emotional states; and (5) NonReacting - the ability to experience unpleasant inner phenomena without engaging in maladaptive behaviour. Investigation of her scores on these five facets of mindfulness revealed moderate improvement during the intervention when the subject was practicing the mindfulness techniques. However, these scores declined over the follow up period when she discontinued the practice, leading the authors to suggest that she was at risk of relapse.

Taken together, the findings of these two case studies suggest that mindfulness-based psychotherapy may be useful in the treatment of problem gambling so long as participants engage in the practice of mindfulness. However, considering the limitations of case studies, randomised controlled trials are required to confirm any conclusions about the efficacy of mindfulness-based psychotherapy for problem gambling.

\section{Models of Mindfulness in Context of Problem Gambling}

Several theoretical models have been proposed to explain how mindfulness operates. These are the Differential Activation Hypothesis (DAH; Lau, Segal, \& Williams, 2004), the Self-Regulatory Executive Function (S-REF) model of emotional disorder (Wells \& Matthews, 1994, 1996), and the Intention Attention Attitude model (IAA; Shapiro, Carlson, Astin, \& Freedman, 2006). The following discussion considers these models in relation to problem gambling.

\section{Differential Activation Hypothesis}

The DAH proposes that individuals previously diagnosed with depression are vulnerable to depressive relapse through automatic activation of negative mood states that result in a downward spiral of negative cognitions. MBCT was developed to interrupt this 
process by assisting individuals to shift from a 'doing' mode of mind to a 'being' mode of mind through mindfulness training (Segal et al., 2002). The 'doing' mode is characterised by an attempt to shift from a current state of dissatisfaction to a more ideal state of affairs. If this shift cannot be achieved, then negative feelings are experienced and dysfunctional coping behaviours such as gambling may be initiated to reduce the discrepancy between these modes of mind.

The DAH may be applied to problem gambling in several ways. For example, negative feelings may be automatically triggered after losing money and habitual patterns of thinking invoked in a vain attempt to restore the loss. In contrast, the 'being' mode is characterised by a state of mind where thoughts and feelings are recognised as objects that arise, become subject of awareness like any other present moment experience, and then disappear. Segal et al. (2002) liken this to a 'decentring' of mental experience, which Shapiro et al., 2006) also refer to as 'reperceiving'. That is, the individual gains the ability to step outside of their current experience, thereby changing their relationship with it (Safran \& Segal, 1990). Through adoption of a being mode, gamblers are placed in a position whereby the experience of any cognition that may precipitate or maintain gambling behaviour can be recognised and simply noted without the need for further action.

Self-Regulatory Executive Function model of Emotional Disorder

The S-REF model provides additional explanatory power for this process by offering a multi-level architecture within which information processing is supported by the interaction of three levels of cognition (Wells, 2002). The first is a level of stored knowledge or beliefs that are held in long term memory (beliefs are conceptualised as a meta-cognitive plan that guides the activities of the on-line processing system), the second is a level of on-line processing that supports the appraisal and execution of coping strategies that are reliant upon attention, and the third is a lower level of reflexive processing that operates outside of 
awareness (Wells, 2002). According to this model, gambling behaviour can be viewed as the end result of the interaction between stored knowledge and beliefs, on-line processing in relation to the interpretation of events (such as encountering a gambling venue or interpretation of the pattern of wins and losses generated while gambling), and a lower level of processing operating outside awareness that drives underlying feelings and urges associated with gambling activity.

Overarching this process are two modes of cognitive processing - the object mode and the meta-cognitive mode (Wells, 2002). Gamblers with a low level of mindfulness would operate in the object mode, whereby the interaction of these three levels of cognition would result in a perceived reality that must be acted upon. Given a high risk situation, gambling behaviour would be automatically initiated followed by a subsequent strengthening of such cognitions. Alternatively, those with a high degree of mindfulness operate in the metacognitive mode. In this case, the interactions between these three levels of cognition would result in the realisation that mental events are subjective and simply come and go. In recognising mental events as impermanent, gamblers operating in this mode would be placed in a much better position to evaluate mental events as they arise and initiate meta-cognitive control behaviours such as redirecting attention. With practice, this process offers the potential to restructure maladaptive cognitions and facilitate the development of more functional responses.

\section{Intention Attention Attitude Model}

While no model offers a definitive explanation as to how mindfulness operates, the IAA model compliments the earlier DAH and S-REF models by offering a tri-axiomatic model of mindfulness that is most consistent with the construct as it is currently understood. In this context, the IAA model conceptualises mindfulness as three interwoven components of intention, attention and attitude that occur simultaneously within a single cyclical process 
(Shapiro et al., 2006). That is, intentionally observing the mind and body, attending to present moment experiences, with an attitude of acceptance. Shapiro et al. refer to these components as axioms since they are "the fundamental building blocks out of which other things grow" (p. 375).

For problem gamblers seeking to control or abstain from gambling, intention can be seen as the intention to begin and sustain a mindfulness practice with the aim of doing something about their problematic gambling behaviour. This vision is a dynamic and evolving process whereby the intention to sustain a regular mindfulness practice may move gamblers from the intention to regulate their gambling behaviour, to one of embarking on a journey of self-exploration, and which may finally culminate in self-liberation (Shapiro et al., 2006). The second axiom is attention, which involves paying attention to gambling-related thoughts and feelings in the present moment (Toneatto et al, 2007). Rather than challenging the content of thoughts and feelings, attention involves attending to events as they occur without the need to change anything. This leads to the third axiom which is attitude. The attitude that is brought to bear on whatever is being attended to is crucial. If the object of attention is judged as either good or bad, then an aversion or attraction to the object develops. This can become an issue for problem gamblers since factors such as intolerance of boredom, sensation seeking, avoidance of stress, or dysphoric mood all may contribute to a greater desire to engage in gambling behaviour (Sharpe, 2002). In developing an accepting attitude to the object attended to, gamblers can simply observe such states come and go rather than develop an attachment to the behaviours previously elicited by them.

The fundamental rationale for mindfulness-based psychotherapy for problem gambling is to teach problem gamblers to attend, with an attitude of discovery, observation and dispassionate awareness, to thoughts and feelings that may lead to gambling behaviour (Toneatto et al., 2007). The interaction of intention, attention and attitude as described in the 
IAA model of mindfulness can be readily discerned in this description of the therapy. By fostering the development of these fundamental building blocks, mindfulness training may offer a way for problem gamblers to break out of the cognitive loop that develops from repeated associations between gambling, interpretation of the pattern of wins/losses, and the strengthening of gambling-related urges and desires by helping them to experience their cognitions without identifying with them or clinging on to them in any way.

\section{Mechanisms of Mindfulness}

While these models provide an interesting theoretical framework for explaining how mindfulness may operate, the mechanisms by which mindfulness training may improve therapeutic outcomes remains a topic of speculation (Bishop, 2002; Bishop et al., 2004; Carmody, Baer, Lykins, \& Olendzki, 2009). Mindfulness training involves acquisition of skills necessary to watch cognitions come and go and respond when noticed with reduced conviction as to their validity (Toneatto et al., 2007). The ability to experience cognitions in this manner has important implications given the apparent degree of automaticity of gambling-related cognitions (Boyer \& Dickerson, 2003; McCusker \& Gettings, 1997). Toneatto et al. (2007) argued that mindfulness practice facilitated behavioural change by modifying the relationship problem gamblers have with their gambling-related cognitions. This is achieved by reducing the degree of attachment to 'the seeming truth of their erroneous gambling-related perceptions', thereby assisting problem gamblers to react less automatically to habitual patterns of thinking.

However, mindfully attending to present moment experience has been conceived as a meta-mechanism of action which overarches other direct mechanisms associated with positive change (Shapiro et al., 2006). Overconfidence and risk willingness, myopic focus on reward, mechanisms related to ego-involvement, and mechanisms related to psychological distress are suggested to act as mechanisms of action in the inverse relationship between 
dispositional mindfulness and problem gambling (Baer et al., 2006, Bowen, Witkiewitz, Dillworth, \& Marlatt, 2007; Carmody et al., 2009; Coffey \& Hartman, 2008; de Lisle et al., 2011b; Lakey et al., 2007).

\section{Overconfidence and Risk Willingness}

Lakey et al. (2007) found that the relationship between dispositional mindfulness and problem gambling was mediated by cognitive processes which underlie gambling-related risk-taking and decision-making tasks. These were assessed by the Georgia Gambling Task (Goodie, 2003) which measures overconfidence and risk-willingness. As such, problem gamblers with a higher level of dispositional mindfulness displayed greater accuracy in assessing gambling-related risk taking than less mindful gamblers. Lakey et al. speculated that a higher degree of mindfulness may help problem gamblers inhibit distraction from intrusive thoughts, thereby helping them to more effectively process risk relevant stimuli and reduce the level of bet acceptance.

\section{Myopic Focus on Reward}

Lakey et al. (2007) also found that performance on the Iowa Gambling Task (IGT; Bechara, Damasio, Damasio, \& Anderson, 1994), which measures myopic focus on reward, partially mediated the relationship between mindfulness and problem gambling severity. As such, gamblers with a higher level of dispositional mindfulness could learn mixed reward and punishment contingencies better than less mindful gamblers. Lakey et al. suggested that a heightened degree of mindfulness may allow for better recognition of affective states caused by risk taking. This may help more mindful gamblers to be better attuned to the potential for loss rather than remain focussed on the potential for reward. In this way, Lakey et al. argued that greater dispositional mindfulness may temper impulsive risk taking when placing bets.

\section{Ego-involvement}


Given that performance on risk-taking tasks only partially mediated the relationship between mindfulness and problem gambling severity, Lakey et al. (2007) suggested that other mechanisms must be involved in this relationship. Problem gambler's ego-involvement in gambling represented another possibility. Some symptoms displayed by problem gamblers, such as gambling pre-occupation, may be an example of the tendency of some problem gamblers to imbue gambling experiences with a heightened degree of egoic attachment to the outcome of these experiences. More mindful problem gamblers may be able to mitigate such egoic-involvement by fostering awareness of these experiences, thereby reducing the degree of attachment to them. As yet, however, no study has investigated whether ego-involvement mediates the relationship between dispositional mindfulness and problem gambling outcomes.

\section{Psychological Distress}

Lakey et al. (2007) also speculated that mechanisms relating to psychological distress mediated the relationship between dispositional mindfulness and problem gambling. There is now substantial evidence that problem gambling is associated with significant personal distress (Dickerson, Baron, Hong, \& Cottrell (1996) and a range of psychiatric disorders, such as mood disorders, anxiety disorders, alcohol and substance dependence, personality disorders, and other impulse control disorders (Lorains, Cowlishaw, \& Thomas, 2011; Petry, 2005; Winters \& Kushner, 2003). This often manifests in terms of major depressive disorders, suicide attempts, and alcohol and substance abuse (Australian Institute of

Gambling Research, 1995). Furthermore, problem gambling may result in different levels and types of harm such as personal, familial, social, vocational, educational, financial and legal difficulties (Productivity Commission, 2010; Rickwood et al., 2010).

Several studies established that an inverse relationship existed between dispositional mindfulness and psychological distress in a range of samples such as university students, 
community adults, and substance users (Arch \& Craske, 2006; Bowen et al., 2007; Carmody et al., 2009; Coffey \& Hartman, 2008; Shapiro et al., 2006). That is, a heightened degree of mindfulness is associated with less psychological distress. This relationship has also been identified in two treatment seeking samples of problem gamblers (de Lisle et al., 2011a). There is an emerging literature that has investigated the role of other psychological processes that mediate the inverse relationship between dispositional mindfulness and psychological distress (Arch \& Craske, 2006; Bowen et al., 2007; Carmody et al., 2009; Coffey \& Hartman, 2008; Shapiro et al., 2006). These additional mechanisms include values clarification; emotional, cognitive, and behavioural flexibility; exposure; non-attachment; emotion dysregulation/distress intolerance; thought suppression; and rumination (Bowen et al. 2007; Carmody et al., 2009; Coffey \& Hartman, 2008; de Lisle et al., 2011b; Shapiro et al., 2006). Of these, emotion dysregulation/distress intolerance, thought suppression, and rumination have been tested in problem gambling samples (de Lisle et al., 2011b). In this study, the tested model posited that rumination, emotional regulation/distress intolerance, and thought suppression mediated the relationship between dispositional mindfulness and psychological distress, and that psychological distress was associated with measures of problem gambling behaviour. However, de Lisle et al. argued that it is equally plausible that these mechanisms also mediate the direct relationship between mindfulness and problem gambling behaviour, whereby increased mindfulness may help gamblers watch gamblingrelated thoughts and feelings come and go without feeling the need to act on them (Toneatto et al., 2007). There is no reason to believe that self-regulation; values clarification; emotional, cognitive, and behavioural flexibility, exposure, and non-attachment would not also explain problem gambling outcomes in addition to psychological distress (Shapiro et al., 2006). The remainder of this article will explore the possible role of these mediators in the development of psychological distress in problem gamblers. 
Values clarification. Values clarification refers to the ability of individuals to recognise what is truly meaningful in their life (Shapiro et al, 2006). For problem gamblers, automatic processing relating to gambling may limit considerations of options that would be more congruent with their needs and values (Brown \& Ryan, 2003). A higher degree of dispositional mindfulness may provide the opportunity to observe their values and reflect upon them with greater objectivity, so that they can rediscover and choose behaviour that is more consistent with their values (Brown \& Ryan, 2003). Although there is evidence that more mindful individuals act in ways that are more congruent with their values and interests (Brown \& Ryan, 2003), there has been no investigation of the role of values clarification in problem gambling. However, Carmody et al. (2009) found that values clarification was a partial mediator in the relationship between a composite mindfulness/ reperceiving variable and psychological symptom reduction. The mindfulness/reperceiving variable was employed because Carmody et al. found no meditational relationship using separate measures of mindfulness and reperceiving. However, values clarification and emotional, cognitive, and behavioural flexibility emerged as partial mediators when mindfulness and reperceiving scores were combined. In doing this, the authors concluded that mindfulness and reperceiving (decentring) are highly overlapping constructs.

Emotional, cognitive, and behavioural flexibility. Emotional, cognitive, and behavioural flexibility is another potential mechanism of mindfulness (Shapiro et al., 2006). For some problem gamblers, gambling represents an automatic, habitual and rigid reflexive pattern of behavioural reactivity to life and emotional stress (Blaszczynski \& Nower, 2002) that may result from conditioning, automaticity, and being overly identified with their current experience (Shapiro et al., 2006). Emotional, cognitive, and behavioural flexibility may therefore represent another mechanism by facilitating greater freedom of choice and more adaptive and less automatic behavioural responses to stressors (Shapiro et al., 2006). There 
has yet to be an investigation of the role of emotional, cognitive, and behavioural flexibility in problem gambling. However, Carmody et al. (2009) found that emotional, cognitive, and behavioural flexibility was a partial mediator in the relationship between a composite mindfulness/ reperceiving variable and psychological symptom reduction.

Exposure. Exposure has been successfully used in the treatment of many disorders by desensitising individuals to distressing psychological states (Shapiro et al, 2006).

Mindfulness operates in a similar manner by promoting awareness and acceptance of distressing psychological states, thereby lessening the intensity of distressing thoughts and feelings over time. This process may help problem gamblers experience strong emotions with greater objectivity and less reactivity though learning that emotions, thoughts, or body sensations are not so overwhelming or frightening (Shapiro et al., 2006). The degree to which mindfulness skills can lead to the ability to experience strong emotions or gambling urges without excessive reactivity in problem gamblers has yet to be explored. However, current indications are that exposure does not mediate the relationship between a composite mindfulness/ reperceiving variable and psychological symptom reduction (Carmody et al., 2009).

Non-attachment. Coffey and Hartman (2008) found that non-attachment is a mediator in the inverse relationship between dispositional mindfulness and psychological distress. Individuals tend to avoid negative emotions and become attracted to positive ones. When an individual places external situational requirements on such experiences, then an attachment to that situation may result (McIntosh \& Martin, 1992). For problem gamblers, this may result in an attachment to the outcome of their gambling. However, individuals with a greater level of dispositional mindfulness are more likely to accept current experience just as it is, and are less likely to become attached to the external conditions that may have precipitated such experiences (Brown, Ryan, \& Creswell, 2007). Coffey and Hartman speculated that 
suspension of the tendency to evaluate experience as either positive or negative is incompatible with evaluating one's experience against the external criteria. Another explanation is that direct engagement with inner experience is intrinsically more satisfying than relying on external conditions to be met in order to feel happy (Coffey \& Hartman, 2008). More research is required in order to assess these explanations further and to determine the degree to which non-attachment plays a role in the relationship between mindfulness and problem gambling.

Emotional dysregulation/distress intolerance. Emotional regulation refers to "the process of modulating one or more aspects of an emotional experience or response" (Chambers, Gullone, \& Allen, 2009, p. 564). Emotion regulation is subsumed under the broader category of distress/tolerance, which represents an individual's overall capacity "to experience and withstand negative psychological states" (Simon \& Gaher, 2005, p.83). Brown and Ryan (2003) demonstrated that people who scored higher on a psychometrically sound measure of mindfulness reported significantly greater self-regulated emotion and behaviour. Moreover, brain imaging studies support the hypothesis that mindfulness is effective in regulating affect (Davidson et al., 2003; Lazar et al., 2005). Coffey and Hartman (2008) found that emotion dysregulation mediated the inverse relationship between dispositional mindfulness and psychological distress in two samples of introductory psychology students. Several studies also suggest that mindfulness training improves tolerance for strong impulses associated with substance use (Bowen et al., 2007), borderline personality disorder (Linehan et al., 2006) and binge eating (Kristeller \& Hallett, 1999). Vohs and Baumeister (2004) considered that the ability to regulate impulses in this manner was essential in overcoming impulse control disorders such as problem gambling.

Despite speculation remaining as to how long mindfulness must be practiced in order to achieve a benefit (Arch \& Craske, 2006; Taylor \& Mireault, 2006), mindfulness training is 
thought to assist people cope with strong emotional states (Arch \& Craske, 2006; Hayes \& Feldman, 2004; Linehan et al., 1993a). Arch and Craske (2006) found that a non-clinical sample reported reduced negative affect and greater willingness to view highly negative images after mindfulness training. They concluded that mindfulness training improved the capacity to remain in contact with present moment thoughts and feelings by increasing tolerance of strong emotions. Similarly, Shapiro et al. (2006) considered that mindfulness training allows for the experience of strong emotions with greater objectivity and less reactivity.

Deficits in emotional regulation are strongly implicated in continued problem gambling behaviour (Blaszczynski \& Nower, 2002; Lee, LaBrie, Grant, Kim, \& Shaffer, 2008; Sharpe, 2002; Shead, Callan, \& Hodgins, 2008; Stewart \& Zack, 2008). It has been found that problem gamblers who have strong expectations that gambling augmented positive mood made significantly riskier choices than those gamblers who do not have this expectation (Shead et al., 2008). Moreover, there is substantial evidence that problem gamblers are motivated to gamble in order to reduce negative mood states or to improve positive emotions (Blaszczynski \& Nower, 2002; Sharpe, 2002; Shead et al., 2008; Stewart \& Zack, 2008). For those seeking excitement, this may take the form of horse racing, whereas electronic gaming machine gamblers tend to gamble to avoid life stressors (Blaszczynski \& Nower, 2002; Cocco, Sharpe, \& Blaszczynski, 1995; Sharpe, 2002). In a treatment seeking population of problem gamblers, de Lisle et al. (2011b) found that the inverse relationship between dispositional mindfulness and psychological distress was mediated by attentional deficits in the experience of negative affect. In this way, problem gamblers may learn through mindfulness training to accept strong emotions by mindfully attending to present moment emotional states. This offers the potential to interrupt automatic responses to such states by 
teaching problem gamblers to step back from what is noticed and simply watch strong emotions come and go.

Thought suppression. Thought suppression may represent another mechanism in the inverse relationship between dispositional mindfulness and psychological distress (Coffey \& Hartman, 2008; Bowen et al., 2007). A simple strategy employed by many problem gamblers when attempting to discontinue gambling is to suppress gambling-related thoughts (Ciarrocchi, 2002). However, thought suppression is known to paradoxically increase the frequency and intensity of unwanted thoughts (Wegner, 1989; Wegner \& Erber, 1992). Consequently, unwanted thoughts may become more pronounced as individuals attempt to disengage from gambling.

Thought suppression has been considered an important factor in the maintenance of a wide range of clinical disorders (Najmi \& Wegner, 2008; Rassin, 2005). Addictive behaviour research has demonstrated that thought suppression can impede attempts to discontinue smoking (Toll, Sobell, Wagner, \& Sobell, 2001) and alcohol use (Palfai, Monti, Colby, \& Rohsenow, 1997). Similarly, participation in a mindfulness meditation course was found to result in greater decreases in attempts to avoid unwanted thoughts relating to alcohol use when compared to a control group (Bowen et al., 2007). Bowen et al. concluded that the decrease in thought suppression partially mediated the effect of mindfulness practice on postcourse alcohol use.

Coffey and Hartman (2008) found that thought suppression mediated the inverse relationship between dispositional mindfulness and psychological distress in two samples of introductory psychology students. The potential of mindfulness-based interventions to foster awareness and acceptance of intrusive thoughts represents a powerful strategy for dealing with behavioural problems (Kavanagh, Andrade, \& May, 2004). While empirical evidence in relation to the problem gambling population is limited, de Lisle et al. (2011b) found 
significant negative relationships between dispositional mindfulness, thought suppression, and psychological distress.

Rumination. There is clear empirical support for the role of mindfulness in reducing ruminative thought generally (Borders, Earleywine, \& Jajodia, 2010; Jain et al., 2007; Ramel, Goldin, Camona, \& McQuaid, 2004; Shapiro, Brown, \& Biegel, 2007). According to Segal et al., (2002), rumination involves a judgement and an evaluation about current experience. When such judgments result in attachment to an object or outcome, psychological well-being is negatively impacted (McIntosh \& Martin, 1992). Shapiro et al. (2006) considered that mindfulness permits individuals to see the present moment as it is without judgment and to respond rather than react according to prior habit, conditioning or experience.

Rumination has been identified as a mediator of the relationship between mindfulness and psychological distress in two samples of introductory psychology students (Coffey \& Hartman, 2008). Mindfulness training has also been negatively correlated with rumination (Kumar, Feldman, \& Hayes, 2008). Other research has established a significant relationship between dysphoric affect and automatic ruminative thought patterns in those at risk of relapse from depression (Teasdale, Segal, \& Williams, 1995).

Given the high rates of co-morbidity and distress associated with problem gambling, it is reasonable to assume that problem gambling is also associated with automatic ruminative thought patterns. Rumination in problem gambling also involves habitual patterns of thinking directed toward reliving past gambling experiences, planning to gamble, or thinking of ways to secure money with which to gamble (American Psychiatric Association, 2002). Atlas and Peterson (1990) also found that rumination after a loss was in turn associated with larger wagers on subsequent races and a tendency toward less successful wagers. Furthermore, Ratelle, Vallerand, Mageau, Rousseau, and Provencher (2004) showed that gamblers who were obsessive about gambling displayed poorer vitality and concentration in daily tasks, as 
well as increased rumination, anxiety, negative mood, and guilt than those who effectively controlled their gambling.

In exploring the relationship between dispositional mindfulness and psychological distress in problem gamblers, de Lisle et al. (2011b) found tentative evidence for the role of rumination in mediating the inverse relationship between dispositional mindfulness and psychological distress. Therefore, it is likely that mindful problem gamblers are more aware of the ruminative thought patterns associated with the experience of psychological distress. While the small sample size used in the de Lisle et al. study precluded any firm conclusions, it is possible that raising mindfulness may allow problem gamblers to dispassionately observe the tendency to dwell on unwanted thoughts and respond to such ruminations more adaptively. Since problem gamblers demonstrate a strong attachment to gambling behaviour (Orford, Morison, \& Somers, 1996), increasing mindfulness may represent the antithesis of ruminative thought because it is intentional, is focussed on the present moment, and is nonjudgmental (Williams, Teasdale, Segal, \& Kabat-Zinn, 2007).

\section{Proposed Model of Mindfulness Mechanisms in Problem Gambling}

Figure 1 provides a theoretical model of the relationship between mindfulness and problem gambling behaviour based on the previous review of the empirical and theoretical literature. 


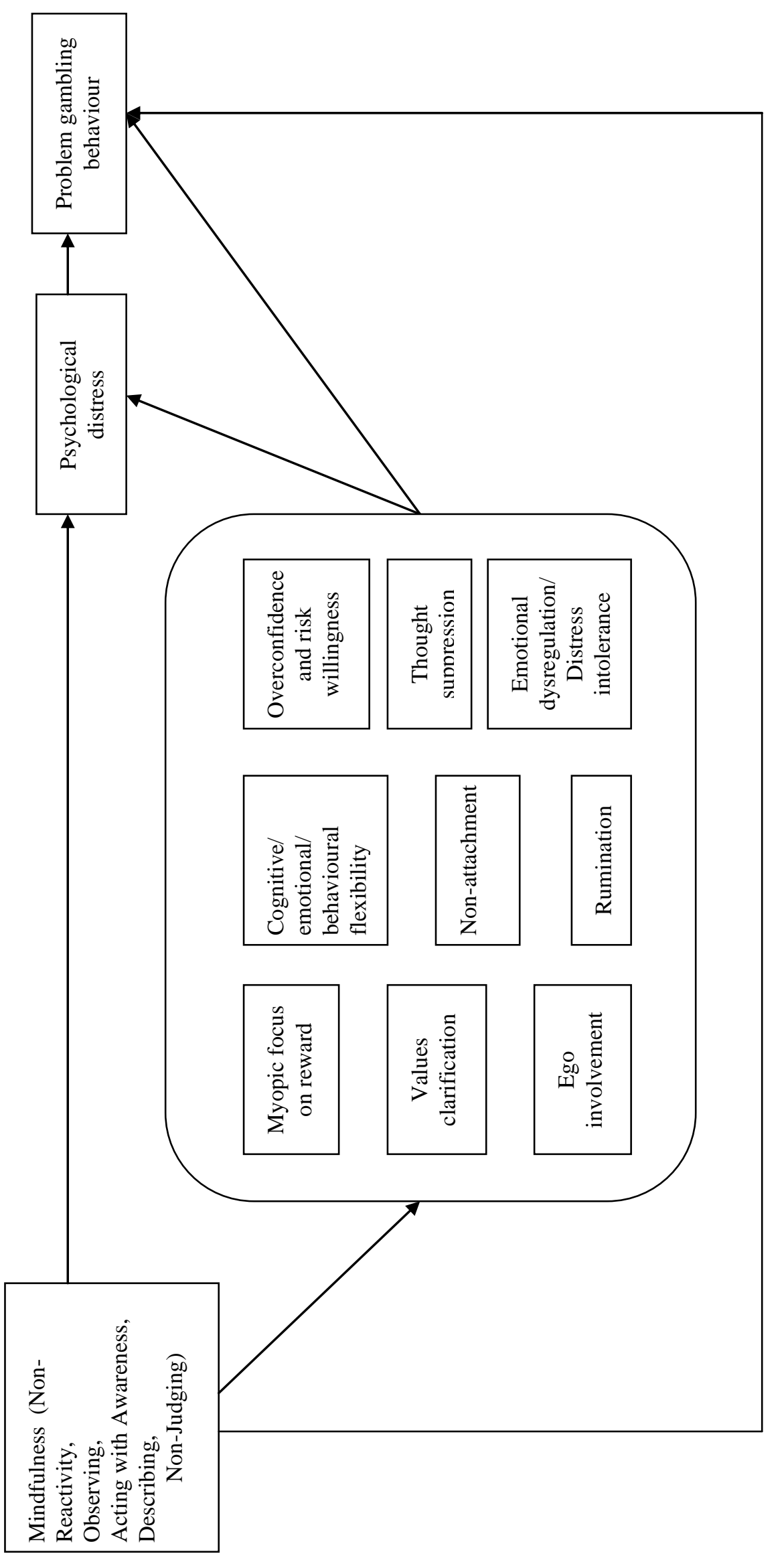

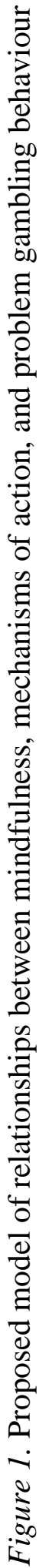


Figure 1 displays the possible mechanisms of action underpinning the relationship between dispositional mindfulness and problem gambling behaviour. Mindful problem gamblers tend to display less severe problem gambling outcomes (de Lisle et al., 2011b; Lakey et al., 2007), which may operate through overconfidence and risk willingness, myopic focus on reward, and ego involvement (Lakey et al., 2007). This relationship also seems to be mediated through lower levels of psychological distress (de Lisle et al., 2011b). The model also highlights that the inverse relationship between dispositional mindfulness and psychological distress may be mediated by values clarification; emotional, cognitive, and behavioural flexibility; non-attachment; emotion dysregulation/distress intolerance; thought suppression; and rumination (Bowen et al., 2007; Carmody et al., 2009; Coffey \& Hartman, 2008; de Lisle et al., 2011b; Shapiro et al., 2006). These mechanisms may also mediate the direct relationship between dispositional mindfulness and problem gambling behaviours, whereby increased mindfulness may help gamblers watch gambling-related thoughts and feelings come and go without feeling the need to act on them (Toneatto et al., 2007).

It is evident that the relationship between mindfulness and problem gambling behaviour is likely to be a very complex one. Mindfulness is a complex construct that should be investigated at a facet level in order to clarify the relationship between the facets and other variables of interest (Baer et al., 2006). Furthermore, the limited number of studies that have investigated the mechanisms of action, methodological limitations such as lack of randomised controlled trials and small sample sizes, lack of suitable assessment instruments, and continued debate as to an operational definition of the mindfulness construct (Carmody et al., 2009) limit understanding as to how mindfulness may improve problem gambling outcomes. Therefore, future studies may wish to explore these relationships in more detail using appropriate measures and statistical procedures with large community and treatment-seeking samples. 


\section{Mindfulness-based Interventions Applicable to Problem Gambling}

There are four mindfulness based approaches that may be appropriate in the treatment of problem gambling. These are Mindfulness-Based Stress Reduction (MBSR; Kabat-Zinn, 2005), Mindfulness-Based Cognitive Therapy (MBCT; Segal et al., 2002), Dialectical Behaviour Therapy (DBT; Linehan, 1993a, 1993b), Acceptance and Commitment Therapy (ACT; Hayes, Strosahl, \& Wilson, 1999), as well as many different variants of these approaches (Baer \& Krietemeyer, 2006). Although different, each approach attempts to operationalise and teach a way of paying attention to present-moment experience that has the potential to reduce symptoms and improve well-being for a wide range of the population (Baer \& Krietemeyer, 2006). While an in-depth discussion of each approach is beyond the scope of this review, each integrates mindfulness within a cognitive-behavioural framework (Lau \& McMain, 2005).

MBSR comprises eight weekly sessions intended for a group setting. It consists of discussion about the nature of stress, its impact on psychological and physiological functioning, instruction on body scanning, and various meditative techniques (Kabat-Zinn, 2005). In a meta-analysis of 64 health-related studies utilising MBSR, Grossman, Niemann, Schmidt and Walach (2004) concluded that MBSR may enhance coping with a broad range of clinical and non-clinical problems. Despite its success, MBSR has not yet been applied to the treatment of problem gambling.

MBCT utilises the framework developed for MBSR and incorporates training in specific cognitive skills for prevention of relapse from depression in a group setting (Segal et al., 2002). Since it was developed, MBCT is now considered of value for heterogeneous groups (Ree \& Craigie, 2007). Furthermore, MBCT incorporates cognitive-behavioural elements previously demonstrated as having positive benefit for problem gamblers, such as psycho-education, pleasure and mastery activities, dealing with difficulty, coping with 
triggers, and relapse prevention (e.g., Dowling, Smith, \& Thomas, 2006, 2007; Dowling, 2009; Petry et al., 2006). There is preliminary support for adaptations of MBCT to other populations and psychiatric disorders, such as binge eating disorder (Baer, Fischer \& Huss, 2005), children with symptoms of anxiety and depression (Semple, Lee, Rosa, \& Miller, 2010), and older adults (Smith, 2006; Smith, Graham, \& Senthinathan, 2007). An adaptation of MBCT has been successfully employed in the treatment of a female problem gambler (de Lisle et al., 2011a).

DBT is primarily used as an intervention for those with borderline personality disorder (Linehan, 1993a, 1993b). DBT is an open ended therapy conducted in individual and group contexts where mindfulness is developed through the application of mindfulness exercises in daily life rather than through formal meditative practices. In comparison, ACT is open-ended and does not require a formal mindfulness practice. ACT is a tailored individual therapy that focuses on developing acceptance of unwanted thoughts and feelings, as well as fostering commitment and action towards living life in accordance with ones values and goals (Harris, 2006). Although ACT has not yet been applied to problem gambling, an adaptation of DBT has been successful in reducing gambling, anger, and substance use in 42 participants with concurrent gambling and anger problems (Korman, Collins, McMain, \& Skinner, 2005). While DBT and ACT are associated with behavioural change, neither of these therapies relies upon the intensive meditative practices advocated in MBSR and MBCT. However, these practices are considered crucial for promoting dispositional mindfulness that all individuals possess.

\section{Limitations of Mindfulness-based Psychotherapy for Problem Gambling}

The key limitation of a purely mindfulness-based intervention for problem gambling is that intensive daily meditative practices are required which may present a significant hurdle for many problem gamblers to overcome (de Lisle et al., 2011a). For example, 
problem gamblers seeking excitement may not be able to tolerate lengthy periods of stillness with no stimulation. However, recent research has demonstrated that meditation experience and home practice of the formal meditation exercises is significantly correlated with the degree of change in facets of mindfulness (Baer et al., 2008; Carmody \& Baer, 2008). Furthermore, changes in mindfulness scale scores have been found to mediate the relationship between meditation practice and positive change (Carmody et al., 2009). Therefore, it appears important that problem gamblers incorporate a mindfulness practice into their daily routine in order to facilitate more adaptive behavioural change.

Shapiro (1992) suggested that the outcome of intensive meditative practices was related to the expectation that subjects had prior to starting a meditative practice. From this perspective, a mindfulness intervention may be most efficacious only when problem gamblers become convinced that the consequences of continuing to gamble are clearly seen to override any benefit derived from continued gambling. The broader mindfulness outcome literature suggests that the resolve to overcome psychological distress may be strengthened by three design features unique to mindfulness-based interventions that emphasise intensive meditative practices (Kabat-Zinn \& Chapman-Waldrop, 1988). The first is that mindfulness practice is presented as a personal challenge that facilitates exploration of a person's potential to improve within a supportive framework. The second is that mindfulness practice offers a way of fostering well-being rather than operating as a series of reductionist techniques designed to achieve an immediate end, such as gambling abstinence. The third is that many different techniques are employed in mindfulness training, such as yoga and a variety of meditation techniques, which increase the possibility that an individual will find a technique that is more congruent with their needs. While there is limited evidence to suggest that a mindfulness intervention may be an acceptable approach for problem gambling (de Lisle et al., 2011a; Toneatto et al., 2007), more research using randomised controlled trials is required 
to definitively demonstrate its efficacy. Since many problem gamblers tend to deny that gambling is a problem, are sceptical of treatment approaches, and attempt to control their behaviour themselves (Tavares, Martins, Zilberman, \& el-Guebaly, 2002), convincing such a population of the therapeutic benefits of a mindfulness practice may be difficult.

To overcome this issue, a more open ended mindfulness therapy such as ACT or DBT may offer a wider scope from which to improve problem gambling outcomes. However, distilling the unique therapeutic impact of the various techniques employed in ACT and DBT may present considerable methodological difficulty. Consequently, the emphasis on intensive meditative practices advocated in inventions such as MBSR and MBCT may provide a better preliminary basis from which to discern the specific role of mindfulness in improving problem gambling outcomes.

Another limitation is that mindfulness may result in adverse consequences for some problem gamblers. While researchers have not extensively investigated potential adverse effects of mindfulness-based interventions, a recent review of the literature revealed tentative evidence for 'altered reality testing', depersonalisation, psychosis, mania, negative affect, exacerbation of depressive symptoms, anxiety, and obsessive rumination after learning to meditate (Melbourne Academic Mindfulness Interest Group, 2006). While current evidence for adverse effects of mindfulness is restricted to case studies and studies with methodological limitations, the exacerbation of such symptoms may be particularly problematic for those problem gamblers with co-morbid psychiatric conditions or reduced cognitive capacity. Given the high likelihood of a co-morbid Axis I or Axis II disorder in problem gambling (Lorains et al., 2011), any intervention that might potentially exacerbate pre-existing psychopathology must be viewed upon with caution. However, since mindfulness increases awareness of internal states, adverse effects may be understandable in the short term. Given that deleterious consequences are possible, the consensus is that 
mindfulness-based approaches should be used with caution and close supervision (Melbourne Academic Mindfulness Interest Group, 2006). This appears particularly relevant for those with a history of psychosis (Chan-Ob \& Boonyanaruthee, 1999; Walsh \& Roche, 1979). As such, it is important that therapists are suitably qualified to deal with unforseen contingencies.

There are also positive indicators for the application of mindfulness-based interventions for problem gambling. There are indications that mindfulness-based psychotherapy is comparable in effectiveness with CBT (Ree \& Craigie, 2007). Mindfulnessbased interventions have also been successfully utilised with individuals with a wide range of presenting issues in the same treatment group (Ree \& Craigie, 2007). This is in contrast to other treatment modalities that may treat different disorders separately (Ree \& Craigie, 2007). As the actual content of what is noticed is unimportant in mindfulness practice, it is irrelevant if the event is symptomatic of depression, anxiety, problem gambling, or any other disorder. As noted earlier, mindfulness-based approaches have been successfully applied to many adult and childhood psychological disorders including psychosis, borderline personality disorder, anxiety, and eating disorders (Baer \& Krietemeyer, 2006). In addition, the approaches are used in the treatment of many medical conditions including chronic pain, fibromyalgia, psoriasis, and cancer (Baer, 2003), and in improving inter-personal relations and stress reduction in the workplace (Baer \& Krietemeyer, 2006). These findings suggest that mindfulness-based interventions may be successful in the treatment of problem gamblers, even if they display co-morbid disorders. However, much more research is required to establish the characteristics of those problem gamblers for whom a mindfulness intervention would be most suited. 
Mindfulness-based interventions may have the potential to improve outcomes for some problem gamblers. However, the paucity of research has precluded the demonstration of efficacy of any mindfulness-based intervention for problem gambling. Toneatto et al. (2007) suggested that mindfulness may be useful either as an adjunct to existing CBT interventions or as a relapse prevention strategy following standard CBT. It is also possible that mindfulness training could be incorporated into existing CBT treatments given that there is some overlap between mindfulness training and some elements of traditional CBT. However, no research has yet investigated these possibilities. It is suggested that future research should initially be focused on existing mindfulness-based approaches given their efficacy for a wide range of presenting issues. This approach would enable evaluation of the unique contribution of mindfulness training to improved therapeutic outcomes (Dimidjian \& Linehan, 2003; Shapiro et al., 2006).

The literature indicates that mindfulness-interventions appear most efficacious where other treatment approaches have failed. For example, MBSR was developed for those with chronic pain who were previously unresponsive to other forms of medical intervention (Grossman et al., 2004; Kabat-Zinn, 2005), MBCT was developed for those who have experienced three or more prior episodes of major depression (Coelho, Canter \& Ernst, 2007; Ma \& Teasdale, 2004), and DBT was developed for borderline personality disorder, a disorder long known to be treatment resistant (Linehan, 1993a, 1993b). Accordingly, it is possible that previously unresponsive problem gamblers may benefit most from a mindfulness-based intervention. However, the significant commitment to a daily mindfulness practice required in interventions such as MBSR and MBCT may make these approaches unpalatable for many gamblers. Future research is required to understand which mindfulness intervention would be most appropriate and for whom. 
Research effort should also be directed to understanding the potential for adverse consequences. Current understanding is restricted to participants who have practiced transcendental meditation rather than those who have attended a formal mindfulness intervention (Melbourne Academic Mindfulness Interest group, 2006). However, clients with a history of psychosis appear particularly at risk. As such, screening of participants prior to any mindfulness intervention is essential in order to assess psychiatric vulnerability. Such screening should be undertaken according to DSM-IV-TR diagnostic criteria using structured clinical interviews (Walker et al., 2006). Risk mitigation can only be performed by suitably qualified professionals. Such qualifications should also extend to appropriate training in the mindfulness intervention to be delivered. While training requirements for the various mindfulness interventions vary, accreditation in MBSR and MBCT require facilitators to adopt a personal mindfulness practice of their own. DBT and ACT accreditation is not as stringent in this regard. Current consensus is that mindfulness interventions should be provided by suitably qualified instructors with the skills necessary to deal with possible adverse reactions (Melbourne Academic Mindfulness Interest group, 2006).

While the number of randomised controlled trails of mindfulness interventions is increasing, there still remain many unanswered questions with respect to this approach (Bishop, 2002). The most fundamental of these is 'what is mindfulness?' and 'what are its mechanisms of action?' Potential mechanisms include overconfidence and risk willingness, myopic focus on reward, ego involvement, lower levels of psychological distress; selfregulation; values clarification; emotional, cognitive and behavioural flexibility; exposure; non-attachment, emotion dysregulation and distress intolerance; reduced thought suppression, and reduced rumination (Baer et al., 2006, Bowen et al., 2007; Carmody et al., 2009; Coffey \& Hartman, 2008; de Lisle et al., 2011b; Lakey et al., 2007). Research efforts have not clearly delineated the mechanisms of action with only partial support for the meditational 
aspects of these variables between mindfulness and psychological well-being (Carmody et al., 2009). Clearly, far more research is required to determine the role of these mechanisms and others in promoting psychological well-being and problem gambling outcomes. Unfortunately, there still exists a lack of psychometrically sound measures that assess these variables (Carmody et al. 2009, Dimidjian \& Linehan, 2003). Moreover, continued debate as to the mindfulness construct continues to hamper mindfulness efficacy investigations.

In summary, this review has highlighted that mindfulness-based interventions for problem gambling is worthy of further exploration, but that they should be considered with some caution. Despite tentative evidence that mindfulness may improve problem gambling outcomes and psychological distress, the existence of non-randomised trials without suitable comparison groups are insufficient to establish the efficacy of any mindfulness-based treatment (Chambless \& Ollendick, 2001). However, evidence is growing that mindfulness interventions have potential in improving outcomes for a wide range of presenting issues. Further research using randomised controlled methodologies is required to evaluate the degree to which mindfulness-based interventions improve therapeutic outcomes for problem gambling. 


\section{References}

American Psychiatric Association. (2000). Diagnostic and Statistical Manual of Mental Disorders, Text Revision (4th ed.). Arlington, VA: Author.

Arch, J. J., \& Craske, M. G. (2006). Mechanisms of mindfulness: Emotion regulation following a focussed breathing induction. Behaviour Research and Therapy, 44, 1849-1858.

Atlas, G. D., \& Peterson, C. (1990). Explanatory style and gambling: How pessimists respond to losing wagers. Behaviour Research and Therapy, 28(6), 523-529.

Australian Institute of Gambling Research. (1995). A critical analysis of two national prevalence studies: The case for alternative strategies. Sydney: Macarthur University of Western Sydney.

Baer, R. A. (2003). Mindfulness training as a clinical intervention - a conceptual and empirical review. Clinical Psychology Science and Practice, 10, 125-143.

Baer, R. A., Fischer, S., \& Huss, D. B. (2005). Mindfulness and acceptance in the treatment of disordered eating. Journal of Rational-Emotive \& Cognitive Behavior Therapy, 23(4), 281-300.

Baer, R. A., \& Krietemeyer, J. (2006). Overview of mindfulness and acceptance-based treatment approaches. In R. Baer (Ed.), Mindfulness-Based Treatment Approaches: A Clinicians Guide to Evidence Base and Applications (pp. 3-27). London: Academic Press.

Baer, R. A., Smith, G. T., Hopkins, J., Krietemeyer, J., \& Toney, L. (2006). Using self-report methods to explore facets of mindfulness. Assessment, 13(1), 27-45.

Baer, R.A., Smith, G.T., Lykins, E., Button, D., Krietemeyer, J., Sauer, S., et al. (2008). Construct validity of the Five Facet Mindfulness Questionnaire in meditating and 
nonmeditating samples. Assessment, 15, 329-342.

Bechara, A., Damasio, A. R., Damasio, H., \& Anderson, S. W. (1994). Insensitivity to future consequences following damage human prefrontal cortex. Cognition, 50, 7-15.

Bishop, S. R. (2002). What do we really know about mindfulness-based stress reduction? Psychosomatic Medicine, 64, 71-84.

Bishop, S. R., Lau, M., Shapiro, S., Carlson, L., Anderson, N. D., Carmody, J., et al. (2004). Mindfulness: A proposed operational definition. Clinical Psychology: Science and Practice, 11(3), 230-241.

Blaszczynski, A., \& Nower, L. (2002). A pathways model for problem and pathological gambling. Addiction, 97(5), 487-499.

Borders, A., Earleywine, M., \& Jajodia, A. (2010). Could mindfulness decrease anger, hostility, and aggression by decreasing rumination? Aggressive Behavior, 36, 28-44.

Bowen, S., Witkiewitz, K., Dillworth, T. M., \& Marlatt, G. A. (2007). The role of thought suppression in the relationship between mindfulness meditation and alcohol use. Addictive Behaviors, 32, 2324-2328.

Boyer, M., \& Dickerson, M. (2003). Attentional bias and addictive behaviour: automaticity in a gambling-specific modified Stroop task. Addiction, 98(1), 61-70.

Brown, K. W, \& Ryan, R. M. (2003). The benefits of being present: Mindfulness and its role in psychological well-being. Journal of Personality and Social Psychology, 84(4), 822-848.

Brown, K. W, \& Ryan, R. M., \& Creswell, J. D. (2007). Mindfulness: Theoretical foundations and evidence for salutary effects. Psychological Inquiry, 18(4), 211-237.

Carmody, J., \& Baer, R. A. (2008). Relationships between mindfulness practice and levels of mindfulness, medical and psychological symptoms and well-being in a mindfulnessbased stress reduction program. Journal of Behavioral Medicine, 31, 23-33. 
Carmody, J., Baer, R. A., Lykins, E. L. B., \& Olendzki, N. (2009). An empirical study of the mechanisms of mindfulness in a mindfulness-based stress reduction program. Journal of Clinical Psychology, 65, 613-626.

Chambers, R., Gullone, E., \& Allen, N. B. (2009). Mindful emotion regulation: An integrative review. Clinical Psychology Review, 29, 560-572.

Chambless, D. L., \& Ollendick, T. H. (2001). Empirically supported psychological interventions: Controversies and evidence. Annual Review of Psychology, 52, 685716.

Chan-Ob, T., \& Boonyanaruthee, V. (1999). Meditation in association with psychosis. Journal of the Medical Association of Thailand, 82, 925-930.

Ciarrocchi, J. W. (2002). Counseling problem gamblers: A self regulation manual for individual and family therapy. San Diego: Academic Press.

Cocco, N., Sharpe, L., \& Blaszczynski, A. P. (1995). Differences in preferred level of arousal in two sub-groups of problem gamblers: A preliminary report. Journal of Gambling Studies, 11, 221-229.

Coelho, H. F., Canter, P. H., \& Ernst, E. (2007). Mindfulness-based cognitive therapy: Evaluating current evidence and informing future research. Journal of Consulting Clinical Psychology, 75(6), 1000-1005.

Coffey, K. A., \& Hartman, M. (2008). Mechanisms of action in the inverse relationship between mindfulness and psychological distress. Complementary Health Practice Review, 13(2), 79-91.

Davidson, R. J., Kabat-Zinn, J., Schumacher, J., Rosenkrantz, M., Muller, D., Santoreli, S. F., et al. (2003). Alterations in brain and immune function produced by mindfulness meditation. Psychosomatic Medicine, 65, 564-570. 
de Lisle, S. , Dowling, N. \&, Allen, J. (2011a). Mindfulness-based cognitive therapy for problem gambling. Clinical Case Studies. Prepublished March 7, 2011, DOI:10.1177/1534650111401016

de Lisle, S. , Dowling, N. \&, Allen, J. (2011b). Mechanisms of action in the inverse relationship between dispositional mindfulness and problem gambling. Manuscript submitted for publication.

Dickerson, M., Baron, E., Hong, S-M., \& Cottrell, D. (1996). Estimating the extent and degree of gambling related problems in the Australian population: A national survey. Journal of Gambling Studies, 12(2), 161-178.

Dimidjian, S. D., \& Linehan, M. M. (2003). Mindfulness practice. In W. O’Donohue, J. Fisher, \& S. Hayes (Eds.), Cognitive behavior therapy: Applying empirically supported techniques in your practice. (pp. 229-237). New York: Wiley.

Dowling, N., Smith, D., \& Thomas, T. (2006). Treatment of female pathological gambling: Efficacy of a cognitive-behavioural approach. Journal of Gambling Studies, 22, 355372.

Dowling, N., Smith, D., \& Thomas, T. (2007). A comparison of individual and group cognitive behavioral treatment for female pathological gambling. Behaviour Research and Therapy, 45, 2192-2202.

Dowling, N., Smith, D., \& Thomas, T. (2009). A preliminary investigation of abstinence and controlled gambling as self-selected goals of treatment for female pathological gambling. Journal of Gambling Studies, 25(2), 201-214.

Goodie, A. S. (2003). The effect of control on betting: Paradoxical betting on items of high confidence with low value. Journal of Experimental Psychology: Learning, Memory, Cognition, 29, 598-610. 
Grossman, P., Niemann, L., Schmidt, S., \& Walach, H. (2004). Mindfulness-based stress reduction and health benefits: A meta-analysis. Journal of Psychosomatic Research $57,35-43$.

Hayes, S. C., \& Feldman, G. (2004). Clarifying the construct of mindfulness in the context of emotion regulation and the process of change in therapy. Clinical Psychology: Science and Practice, 11(3), 255-262.

Hayes, S. C., Follette, V. M., \& Linehan, M. M. (Eds.). (2004). Mindfulness and acceptance: Expanding the cognitive tradition. New York: Guilford Press.

Hayes, S. C., Strosahl, K., \& Wilson, K. G. (1999). Acceptance and Commitment Therapy. New York: Guilford Press.

Harris, R. (2006). Embracing your demons: An overview of Acceptance and Commitment Therapy. Psychotherapy in Australia, 12(4), 2-8.

Jain, S., Shapiro, S. L., Swanick, S., Roesch, S. C., Mills, P. J., Bell, I., et al. (2007). A randomized controlled trial of mindfulness meditation versus relaxation training: Effects on distress, positive states of mind, rumination, and distraction. Annals of Behavioral Medicine, 33, 11-21.

Joukhador, J., Maccallum, F., \& Blaszczynski, A. (2003). Differences in cognitive distortions between problem and social gamblers. Psychological Reports, 92, 1203-1214.

Kabat-Zinn, J. (2005). Full Catastrophe Living - Using the Wisdom of your Body and Mind to face Stress, Pain, and Illness ( $3^{\text {rd }}$ ed.). New York: Bantam Dell.

Kabat-Zinn, J., \& Chapman-Waldrop, A. (1988). Compliance with an outpatient stressreduction program: Rates and predictors of program completion. Journal of Behavioral Medicine, 11(4), 333-352.

Kavanagh, D. J., Andrade, J., \& May, J. (2004). Beating the urge: Implications of research into substance-related desires. Addictive Behaviors, 29, 1359-1372. 
Korman, L., Collins, J., McMain, S., \& Skinner, W. (2005). Concurrent Gambling, Substance Use and Anger: Development of a Brief Integrated Treatment. Ontario: Ontario Problem Gambling Research Centre.

Krasner, M. (2004). Mindfulness-based interventions: A coming of age? Families, Systems, and Health, 22, 207-212.

Kristeller, J. L., \& Hallett, C. B. (1999). An exploratory study of a meditation-based intervention for binge eating disorder. Journal of Health Psychology, 4, 357-363.

Kumar, S., Feldman, G., \& Hayes, S. C. (2008). Changes in mindfulness and emotion regulation in an exposure-based cognitive therapy for depression. Cognitive Therapy and Research, 32, 734-744.

Ladouceur, R., Sylvain, C., Boutin, C., \& Doucet, C. (1998). Understanding and Treating the Pathological Gambler. Chichester: John Wiley \& Sons, Ltd.

Lakey, C. E., Campbell, W. K., Brown, K. W., \& Goodie, A. S. (2007). Dispositional mindfulness as a predictor of the severity of gambling outcomes. Personality and Individual Differences, 43, 1698-1710.

Lau, M. A., \& McMain, S. F. (2005). Integrating mindfulness meditation with cognitive and behavioural therapies: The challenge of combining acceptance- and change-based strategies. Canadian Journal of Psychiatry, 50(13), 863-869.

Lau, M., Segal, Z. V., \& Williams, J. M. G. (2004). Teasdale's differential activation hypothesis: Implications for mechanisms of depressive relapse and suicidal behaviour. Behaviour Research and Therapy, 42, 1001-1017.

Lazar, S. W., Kerr, C. E., Wasserman, R. H., Gray, J. R., Greve, D. N., Treadway, M. T., et al. (2005). Meditation experience is associated with increased cortical thickness. Neuroreport, 16, 1893-1897. 
Lee, T. K., LaBrie, R. A., Grant, J. E., Kim, S. W., \& Shaffer, H. J. (2008). The structure of pathological gambling among Korean gamblers: A cluster and factor analysis of clinical and demographic characteristics. International Journal of Mental Health and Addiction, 6, 551-563.

Lesieur, H. R., \& Heineman, M. (1988). Pathological gambling among youthful multiple substance abusers in a therapeutic community. British Journal of Addiction, 83, 765771.

Linehan, M. M. (1993a). Cognitive-Behavioural Treatment of Borderline Personality Disorder. New York: Guilford Press.

Linehan, M. M. (1993b). Skill Training Manual for Treating Borderline Personality Disorder. New York: Guilford Press.

Linehan, M. M., Comtois, K. A., Murray, A. M., Brown, M. Z., Gallop, R. J., Heard, H. L., et al. (2006). Two-year randomized controlled trial and follow-up of dialectal behavior therapy vs. therapy by experts for suicidal behaviors and borderline personality disorder. Archives of General Psychiatry, 63, 757-766.

López Viets, V. C., \& Miller, W. R. (1997). Treatment approaches for pathological gamblers. Clinical Psychology Review, 17, 689-702.

Lorains, F. K., Cowlishaw, S., Thomas, S. (2011). Prevalence of comorbid disorders in problem and pathological gambling: Systematic review and meta-analysis of population surveys. Manuscript submitted for publication.

Ma, S. H., \& Teasdale, J. D. (2004). Mindfulness-based cognitive therapy for depression: Replication and exploration of differential relapse prevention effects. Journal of Consulting and Clinical Psychology, 72(1), 31-40. 
McCusker, C. G., \& Gettings, B. (1997). Automaticity of cognitive biases in addictive behaviours: further evidence with gamblers. British Journal of Clinical Psychology, $36(4), 543-554$.

McIntosh W. D., \& Martin L. L. (1992). The cybernetics of happiness: The relation between goal attainment, rumination, and affect. In M. S. Clark (Ed.). Review of personality and social psychology Vol. 14. (pp. 222-246). Newbury Park: Sage Publications.

Melbourne Academic Mindfulness Interest Group. (2006). Mindfulness-based psychotherapies: A review of conceptual foundations, empirical evidence and practical considerations. Australian and New Zealand Journal of Psychiatry, 40, 285294.

Najmi, S., \& Wegner, D. M. (2008). Thought suppression and psychopathology. In A. J. Elliot (Ed.), Handbook of approach and avoidance motivation (pp.447-459). New York: Psychology Press.

Orford, J., Morison, V. \& Somers, M. (1996). Drinking and gambling: A comparison with implications for theories of addiction. Drug and Alcohol Review, 15, 47-56.

Palfai, T. P., Monti, P. M., Colby, S. M., \& Rohsenow, D. J. (1997). Effects of suppressing the urge to drink on the accessibility of alcohol outcome expectancies. Behavior Research and Therapy, 35(1), 59-65.

Petry, N. M. (2005). Pathological gambling: Etiology, comorbidity, and treatment. Washington, D.C: American Psychological Association.

Petry, N. M., Ammerman, Y., Bohl, J., Doersch, A., Gay, H., Kadden, R., et al. (2006). Cognitive-behavioral therapy for pathological gamblers. Journal of Consulting and Clinical Psychology, 74(3), 555-567. 
Problem Gambling Research and Treatment Centre. (2011). Guideline for screening, assessment and treatment in problem and pathological gambling. Melbourne: Monash University.

Productivity Commission (2010). Gambling. Productivity Commission Inquiry Report. Volume 1. Report no. 50. Canberra: Productivity Commission.

Ramel, W., Goldin, P. R., Carmona, P. E., \& McQuaid, J. R. (2004). The effects of mindfulness meditation on cognitive processes and affect in patients with past depression. Cognitive Therapy and Research, 28(4), 433-455.

Rassin, E. (2005). Thought suppression. Oxford: Elsevier.

Ratelle, C. F., Vallerand, R. J., Mageau, G. A., Rousseau, F. L. \& Provencher, P. (2004). When passion leads to problematic outcomes: A look at gambling. Journal of Gambling Studies, 20(2), 105-119.

Ree, M. J., \& Craigie, M. A. (2007). Outcomes following mindfulness-based cognitive therapy in a heterogeneous sample of adult outpatients. Behaviour Change, 24(2), 7086.

Rickwood, D., Blaszczynski, A., Delfabbro, P., Dowling, N., \& Heading, K. (2010). The psychology of gambling. InPsych, 32(6), 11-21.

Safran, J. D., \& Segal, Z. V. (1990). Interpersonal Process in Cognitive Therapy. New York: Basic Books.

Segal, Z. V., Williams, J. M. G., \& Teasdale, J. D. (2002). Mindfulness-Based Cognitive Therapy for Depression: A New Approach to Preventing Relapse. New York: Guilford Press.

Semple, R. J., Lee, J., Rosa, D., \& Miller, L. F. (2010). A randomized trial of mindfulnessbased cognitive therapy for children: Promoting mindful attention to enhance socialemotional resiliency in children. Journal of Child and Family Studies, 19(2), 218-229. 
Shapiro, D. H. (1992). A preliminary study of long term meditators: Goals, effects, religious orientation, cognitions. Journal of Transpersonal Psychology, 24(1), 23-39.

Shapiro, S., Brown, K., \& Biegel, G. (2007). Teaching self-care to caregivers: Effects of Mindfulness-Based Stress Reduction on the mental health of therapists in training. Training and Education in Professional Psychology, 1(2), 105-115.

Shapiro, S. L., Carlson, L. E., Astin, J. A., \& Freedman, B. (2006). Mechanisms of mindfulness. Journal of Clinical Psychology, 62(3), 373-386.

Sharpe, L. (2002). A reformulated cognitive-behavioral model of problem gambling: A biopsychosocial perspective. Clinical Psychology Review, 22(1), 1-25.

Shead, N. W., Callan, M. J., \& Hodgins, D. C. (2008). Probability discounting among gamblers: Differences across problem severity and affect-regulation expectancies. Personality and Individual Differences, 45, 536-541.

Simons, J., \& Gaher, R. (2005). The distress tolerance scale: Development and validation of a self-report measure. Motivation and Emotion, 29, 83-102.

Smith, A. (2006). "Like waking up from a dream": Mindfulness training for older people with anxiety and depression. In R. Baer (Ed.), Mindfulness-Based Treatment Approaches: A Clinicians Guide to Evidence Base and Applications (pp. 191-215). London: Academic Press.

Smith, A., Graham, L., \& Senthinathan, S. (2007). Mindfulness-based cognitive therapy for recurring depression in older people: A qualitative study. Aging \& Mental Health, 11(3), 346-357.

Stewart, S. H. \& Zack, M. (2008). Development and psychometric evaluation of a threedimensional Gambling Motives Questionnaire. Addiction, 103, 1110-1117. 
Tavares, H., Martins, S. S., Zilberman, M. L., \& el-Guebaly, N. (2002). Gamblers seeking treatment: Why haven't they come earlier? Addictive Disorders \& Their Treatment, $1(2), 65-69$.

Taylor, D. G., \& Mireault, G. C. (2008). Mindfulness and self-regulation: A comparison of long-term and short-term meditators. The Journal of Transpersonal Psychology, 40(1), 88-99.

Teasdale J. D., Segal Z. V., \& Williams J. M. G. (1995), How does cognitive therapy prevent depressive relapse and why should attentional control (mindfulness) training help?, Behavioral Research and Therapy, 33, 25-39.

Toll, B. A., Sobell, M. B., Wagner, E. F., \& Sobell, L. C. (2001). The relationship between thought suppression and smoking cessation. Addictive Behaviors, 26, 509-515.

Toneatto, T., Vettese, L., \& Nguyen, L. (2007). The role of mindfulness in the cognitivebehavioural treatment of problem gambling. Journal of Gambling Issues, 19, 91-100.

Vohs, K. D., \& Baumeister, R. F. (2004). Understanding self-regulation. In R. F. Baumeister \& K. D. Vohs (Eds.). Handbook of self-regulation (pp. 1-9). New York: Guilford Press. Walker, M., Toneatto, T., Potenza, M. N., Petry, N., Ladouceur, R., Hodgins, D. C., et al. (2006). A framework for reporting outcomes in problem gambling treatment research: The Banff, Alberta Consensus. Addiction, 101(4), 504-511.

Walsh, R., \& Roche, L. (1979). Precipitation of acute psychotic episodes in intensive meditation in individuals with a history of schizophrenia. American Journal of Psychiatry, 136, 1085-1086.

Washington, F. A. (2006). The relationship among rumination and distraction in pathological gamblers. Dissertation Abstracts International: Section B: The Sciences and Engineering 67(1-B), 573. 
Wegner, D. M. (1989). White bears and other unwanted thoughts: Suppression, obsession, and the psychology of mental control. London: The Guilford Press.

Wegner, D. M., \& Erber, R. (1992). The hyperaccessibility of suppressed thoughts. Journal of Personality and Social Psychology, 63, 903-912.

Wells, A. (2002). GAD, metacognition, and mindfulness: An information processing analysis. Clinical Psychology: Science and Practice, 9(1), 95-100.

Wells, A., \& Matthews, G. (1994). Attention and emotion: A clinical perspective. Hove: Erlbaum.

Wells, A., \& Matthews, G. (1996). Modelling cognition in emotional disorder: The S-REF model. Behaviour Research and Therapy 33, 579-583.

Westphal, J. R. (2008). How well are we helping problem gamblers? An update to the evidence base supporting problem gambling treatment. International Journal of Mental Health and Addiction. 6(2), 249-264.

Williams, J. M. G., \& Swales, M. (2004). The use of mindfulness-based approaches for suicidal patients. Archives of Suicide Research, 8, 315-329.

Williams, M., Teasdale, J., Segal, Z., \& Kabat-Zinn, J. (2007). The Mindful Way through Depression. New York: The Guilford Press.

Winters, K. C., \& Kushner, M. G. (2003). Treatment issues pertaining to pathological gamblers with a comorbid disorder. Journal of Gambling Studies, 19(3), 261-277. 


\section{University Library}

\section{- M M I N E R VA A gateway to Melbourne's research publications}

Minerva Access is the Institutional Repository of The University of Melbourne

\section{Author/s:}

de Lisle, SM;Dowling, NA;Allen, JS

Title:

Mindfulness and Problem Gambling: A Review of the Literature

Date:

2012-12-01

Citation:

de Lisle, S. M., Dowling, N. A. \& Allen, J. S. (2012). Mindfulness and Problem Gambling: A Review of the Literature. JOURNAL OF GAMBLING STUDIES, 28 (4), pp.719-739. https:// doi.org/10.1007/s10899-011-9284-7.

Persistent Link:

http://hdl.handle.net/11343/282900 